\title{
Communicated Somatic Markers Benefit Both the Individual and the Species
}

\author{
Kyle I. Harrington, Megan M. Olsen, and Hava T. Siegelmann
}

\begin{abstract}
We use emotional communication within a predator-prey game to evaluate the tradeoff between socioemotional behavior at individual- and species- scales. In this predator-prey game, individual predators and prey use emotion in their decision making, and communicate their emotional state with neighboring conspecifics. The model of emotion is based upon the somatic marker hypothesis. In comparing individual utility and population dynamics we find emotion is capable of both supporting species and individual gain. We suggest this type of dynamic may provide a mechanism for the emergence of altruistic behavior within a species under individual and/or group selection.
\end{abstract}

\section{INTRODUCTION}

Social neuroscience seeks to explain the neural basis of social interactions and behaviors. Relatedly, affective neuroscience seeks to explain the source of emotional behaviors. The interaction between affect and sociality is a question with origins in the works of Spinoza, James, Darwin, and lately by Damasio. Spinoza raised philosophical issues on this topic that provided the foundation to the scientific field of affective research. The relationship between behavior and emotion was extended by William James in his proposal of the interaction between an individual's physical response and emotional state [18]. The interaction of physical state and emotion was later associated with a neurophysiological basis and named the somatic marker hypothesis [13]. In brief, the somatic marker hypothesis states that the physical and mental experience ("the somatic state") is encoded as marker signals in terms of emotion and feelings, and that these signals are integrated into decision making. In this research we present computational evidence that communicated somatic markers have a particular role in decision making: they promote cooperative behavior. Individuals who use emotions and communicate them within their group simultaneously increase the group's and their individual utility. That is, communicated somatic markers provide an evolutionary bias towards cooperative agents.

The somatic marker hypothesis expresses the role of corporeal-based signals in decision making. The studies leading to the somatic marker hypothesis began with ventromedial lesion which showed that such lesions led to pathologies in decision making, emotion, and feeling. In support of the

KIH, MMO, HTS are with the BINDS lab, University of Massachusetts, Amherst (email:kyleh@cs.brandeis.edu, \{molsen, hava\}@cs.umass.edu). $\mathrm{KIH}$ is also with the DEMO lab, Brandeis University, Waltham, MA, and

This work was supported in part by the NSF grant 0757452 and ONR grant 109-0138R. Any opinions, findings, and conclusions or recommendations expressed in this publication are those of the authors and do not necessarily reflect the views of the National Science Foundation or the Office of Naval Research. somatic marker hypothesis is crystallized as a set of signals which influence decision-making as encodings of "musculoskeletal, visceral, and internal milieu" state which affect decision making. Studies of survival behaviors are proposed as an approach to understand the possible functional origins of somatic markers [13].

Although the origins of somatic markers are not known, emotion and decision-making are known to be influenced by similar brain pathways with overlapping areas such as the amygdala, ventromedial, and orbitofrontal cortex [13], [9], [8]. This overlap provides a further support that decision making, emotions, and social behavior ([1], [7], [24], [3], [10], [7]) interact. Our model incorporates these interactions and computationally/logically demonstrates that somatic markers are capable of positive individual and collective impact.

The evolutionary origin of emotional processing and its interaction with decision making and social behavior has been of interest since the early days of evolutionary theory [14]. Darwin explored questions of emotional expressions within man and animals, highlighting the communicative properties of emotional responses, from the warning cry to the alerting roar. These signals convey information of the signaler's somatic state. However, the communication of signals needs to be explored for both the sender and receiver.

In his seminal work on the ubiquity of emotion recognition in humans, Ekman et al. showed that the historically isolated Fore group of New Guinea had significant similarities in identification of emotion to their Western counterparts [16]. This led to the hypothesis that while some components of emotion are learned during lifetime, there are some features of emotion are inherited. An extensive discussion of the evolution of social behaviors is presented in [2]. Yet, the evolutionary retention of emotion is not only dependent upon the communicative properties of the emotion, but how the emotional signals are used for decision making.

An increasingly common trend is to consider emotions as a mechanism for improving decision making [17], [5], [4], [19], [20]. Many computational studies of emotion and decision making focus upon path planning and navigation. Although the results of these studies show notable improvements in simulation and robots, tasks involving multiple agents generally benefit from communication, which is is largely uncharted territory.

In our initial work we examined emotion and emotional communication in multi-agent systems with stigmergic (indirect) communication and basic decision processes [22], [21]. More recently we have examined the population-scale 
dynamics of systems with individual-to-individual emotional communication [23]. In this paper, we show the benefit of communicated emotion through species- and individuallevel metrics within the model system presented in [23]. We conclude by discussing the emergence of cooperation under certain behavioral conditions within a predator-prey game.

\section{MODEL}

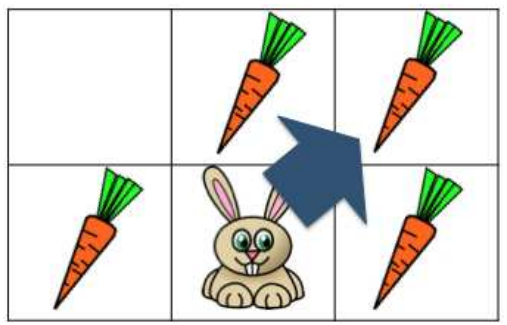

(a) Basic movement is stochastic but biased.

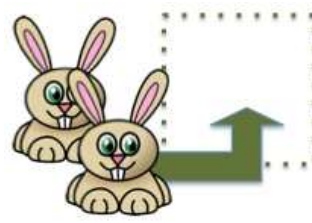

(b) Reproduction parthenogenic.

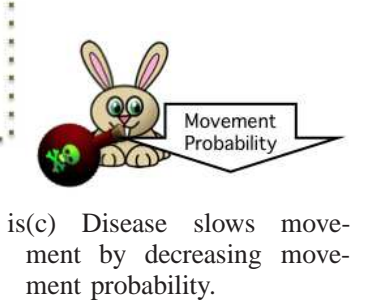

Fig. 1: Mechanics of the basic model.

The basis of the model is the multi-species cellular automata presented in [23]. The simulated world is discretized in a grid with periodic boundary conditions, "wrap-around." Three species may occupy the otherwise empty grid: rabbits, foxes, and carrots. These three species are related via predator-prey interactions. Foxes feed on rabbits, while rabbits feed on carrots. Carrots serve as both an energy input and a vector of disease for the system. All entities breed while mobile entities, rabbits and foxes, move, eat, experience hunger, and suffer from disease.

Reproduction is a primarily time-based process that occurs in carrots, rabbits, and foxes (Fig. 1b). Once a carrot reaches the age of maturity an offspring carrot is produced and moved to a randomly selected adjacent position. Rabbits and foxes reproduce similarly, but switch to a probabilistic breeding mechanism once they reach maturity. However, if there are no open adjacent positions then no breeding is possible. Movement is employed to limit these situations.

Traditional cellular automata utilize deterministic transition rules [25]. Such determinism is infrequent in the face of uncertainty. For this reason we utilize stochastic movement that is biased by preference (Fig. 1a). In the basic model these biases are intuitive. Rabbits move towards carrots and away from foxes, foxes move towards rabbits, while carrots are stationary. Movement bias is computed as a gradient of preference which may be occluded by neighboring conspecifics. The gradient of preference for foxes is computed as

$$
g_{d, f}(t, x, y)=s_{r}(t, x, y)
$$

where direction $d \in\{N W, N, N E, W, E, S W, S\}, t$ is the current time, and $s_{r}(t, x, y)$ indicates the presence or absence of a rabbit at position $(\mathrm{x}, \mathrm{y})$ at the current time with 1 or 0 , respectively. The gradient of preference for rabbits accounts for adjacent predators as well as food

$$
g_{d, r}(t, x, y)=s_{r}(t, x, y)-s_{f}(t, x, y)
$$

where $s_{f}(t, x, y)$ indicates the presence or absence of a fox at position $(\mathrm{x}, \mathrm{y})$ at the current time with 1 or 0 , respectively. The pursuit of food is driven by the movement mechanism even the food may be dangerous.

The transmission of disease begins with the carrot as the source. When a carrot reproduces there is a small probability of the offspring being diseased. It will remain diseased for a constant infection period after which it will have a probability of being cured. If the carrot reproduces before it is cured of the disease it has a higher probability of producing diseased offspring. When a rabbit eats a diseased carrot it follows the same timeline, but causes the rabbit to get hungry twice as fast and move more slowly (Fig. 1c). Disease transfer from rabbit to fox is equivalent with a difference that rabbits do not transfer disease to offspring. Many features from disease to emotion itself contribute to the emotional extension of this model.

\section{A. Emotion and Emotional Communication}

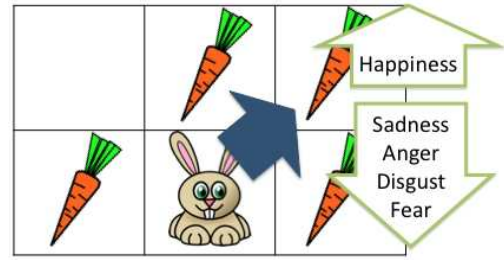

(a) Emotions bias direction of movement.

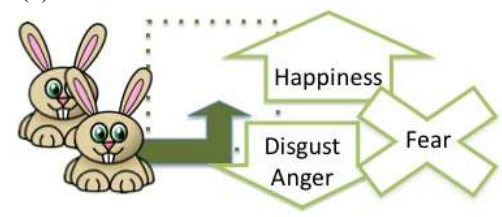

(b) Emotions modulate reproduction.

Fig. 2: Mechanics of model with emotion.

Although there are some low level behaviors that are modulated by emotion, the decision biasing aspect of emotion is derived from observations of relative emotion. For example, an entity gets happier when it eats food. While this will increase the probability of reproduction, it will bias direction movement towards neighboring entities that are happy. This can be thought of as a "the grass is always greener on the other side" bias. Of course, negative emotions act as detractors; "the grass is probably greener in the direction opposite the yellow grass." The non-social effects have been heavily constrained in this model in order to emphasize the communicative properties of emotion. If the reader is familiar with rabbits in the wild she will probably recall walking by 
TABLE I: Emotional experiences for each emotion.

\begin{tabular}{|r|l|}
\hline Emotion & Experience \\
\hline \hline Happiness & 1 if ate food, 0 otherwise \\
\hline Sadness & time since last reproduction \\
\hline Anger & $e^{\text {hunger }}$ \\
\hline Fear (fox) & anger of neighboring foxes \\
\hline Fear (rabbit) & number of neighboring foxes \\
\hline Disgust & 1 if ate diseased food, 0 otherwise \\
\hline Surprise & $\frac{\Sigma_{e} E_{e}(t, x, y)-E_{e}(t-1, x, y)}{5}$ for all emotions except \\
& surprise \\
\hline
\end{tabular}

a multiple rabbits. Upon approaching the closest rabbit it flees, shortly followed by its neighbors. This type of sociobehavioral cue is one of the two primary effects of emotional communication in our model.

Emotions are computed based upon individual experience, which is derived from both internal state, the surroundings, and communicated emotion. The individual's immediate experience is then combined with conspecific communicated emotion and previous emotional state to render the new emotional state. This emotion update is

$$
\begin{array}{r}
E_{e, s p}(t+1, x, y)=\left(1-c_{m, s p}\right) *\left(X_{e, s p}(t, x, y)+\right. \\
\left.c_{c, s p} * C E_{e, s p}(t, x, y)\right)+c_{m, s p} * E_{e, s p}(t, x, y)
\end{array}
$$

where emotion $e \in$ ffear, anger, happiness, sadness, disgust, anger $\}$, species $s p \in$ rabbits, foxes\}, $c_{m, s p}$ is the memory discount, $c_{c, s p}$ is the communication discount, $C E_{e, s p}(t, x, y)$ is the communicated emotion, $X_{e, s p}(t, x, y)$ is the emotional experience present in table $\mathrm{I}$, and $E_{e, s p}(t, x, y)$ is the emotion value. Both communication and memory discounts are within $[0,1]$ such that they serve as geometric discounting factors. The communication discount determines the ratio of the memory that is based upon communicated emotion versus current experience. The memory discount determines how long emotional experiences affect an entity. As was previously mentioned emotions can bias direction of movement (Fig. 2a). The updated gradient of preference equations are

$$
\begin{array}{r}
g_{d, f}(t, x, y)=s_{r}(t, x, y) \\
+\Sigma_{e}\left(v_{e} *\left(E_{e, f}\left(t, x_{d}, y_{d}\right)-E_{e, f}(t, x, y)\right)\right) \\
g_{d, r}(t, x, y)=s_{r}(t, x, y)-s_{f}(t, x, y) \\
+\Sigma_{e}\left(v_{e} *\left(E_{e, r}\left(t, x_{d}, y_{d}\right)-E_{e, r}(t, x, y)\right)\right)
\end{array}
$$

where $v_{e}$ is -1 for $e \in\{$ fear, anger, sadness, disgust $\}, 1$ for $e=$ happiness, and 0 for $e=$ surprise.

Finally, emotions modulate reproduction rates (Fig. 2b). For both rabbits and foxes happiness will increase the reproduction while disgust and anger will decrease it. Rabbits have an additional fear contribution whereby reproduction will not occur if fear surpasses a threshold. The emotion-modulated reproduction rates are

$$
\begin{array}{r}
R(t)=R_{b} *\left(1-R_{b}\right) *\left(E_{\text {happiness }}(t, x, y)\right. \\
\left.-\alpha E_{\text {disgust }}(t, x, y)-(1-\alpha) * E_{\text {anger }}(t, x, y)\right)
\end{array}
$$

where $R_{b}$ is the base probability of reproducing after maturation, and $\alpha$ is the ratio of effect between disgust and emotion. These emotional contributions lead to a number of interesting effects at both the population and individual scales.

\section{INDIVIDUAL UTILITY}

Individual utility measures the benefit of an entity's "somatic state," the amount an entity benefits from its environment. We present two utilities with particularly interesting properties: internal and reproductive. The internal utility is based upon the internal state of each entity, encoding hunger and disease conditions. Both of these conditions are the primary components of an entity's state, aside from the ability to reproduce. However, reproducing is not included in the internal utility function as it does not have direct effects on an entity's life span. The internal utility for foxes and rabbits are measured as seen in Equation 7:

$$
U_{s p}(t, x, y)=\frac{\frac{-h_{s p}(t, x, y)}{\text { starve }_{s p}}-Z_{s p}(t, x, y)+2}{2},
$$

where $h_{s p}(t, x, y)$ is the hunger level, starve $s p$ is the starvation level, and $Z_{s p}(t, x, y)$ is binary and indicates whether a fox or rabbit is diseased.

Reproductive utility describes the internal state of an individual accounting for reproductive behavior as well, as seen in Equation 8:

$W_{s p}(t, x, y)=\frac{-\frac{h_{s p}(t, x, y)}{s t a r v e_{s p}}-Z_{s p}(t, x, y)-\frac{P_{s p}(t, x, y)}{b_{s p}+1}+3}{3}$,

where $P_{s p}(t, x, y)$ is the time since reproduction, and $b_{s p}$ is the time to maturation, with $s p$ representing whether it is a fox or rabbit. Although reproduction occurs probabilistically after maturation the base probability of reproduction in these experiments was 0.75 . The average individual reproductive utility for both rabbits and foxes does not present any distinction between emotional configurations; however, we will see in the results that changes in reproductive utility demonstrate interesting trends.

\section{RESULTS}

Parameter values for the experiments presented in this paper are presented in table II. Evidence of the robustness of this model to changes in parameters can be seen in [23], [21]. In this paper we compare the above two utility functions with the overall group dynamics with and without emotion. This comparison will show whether the use of emotions helps the individual, the group, or both. We will also examine what aspects of survival most influence the success of the individual versus the group by a comparison between the two utilities.

\section{A. Population Dynamics}

An initial study of the population dynamics of this system is presented in [23]. We highlight the differences in rabbit and fox population size under each emotion configuration (Figs. 3a and 3b). The foxes' use of emotion shows lower 
TABLE II: Parameters used in experiments.

\begin{tabular}{|r|r|}
\hline Parameter & Value \\
\hline \hline Number of trials & 10 \\
\hline World size & $50 \times 50$ units \\
\hline Simulation time & 700 steps \\
\hline Initial fox population & 250 foxes \\
\hline Initial rabbit population & 500 rabbits \\
\hline Initial carrot population & 1500 carrots \\
\hline$c_{c, r}$ & 0.75 \\
\hline$c_{c, f}$ & 0.55 \\
\hline$\alpha$ & 0.4 \\
\hline
\end{tabular}

rabbit population sizes, while the configuration in which only rabbits have emotion is indistinguishable from the population size when neither species has emotion. While it is suggested that the reason for the latter effect may be due to a carrying capacity limit on the rabbit population, the detrimental effect of fox emotion on the rabbit population is apparent. Some contention may arise due to the predating relationship of foxes to rabbits inherently decreasing the rabbit population; however, examination of the fox population offers additional insights.

Trends in the fox population are closer to what one would expect to see for a population far enough below their carrying capacity. The population curve in the only fox emotion configuration is consistently above that of the no emotion configuration, and both are above the only rabbit emotion configuration. This is the intuitive result one would expect if emotion does serve a purpose in these types of systems. Additionally, when both species have emotion the population is similar in size to when neither have emotion. Relating back to the rabbit population curves, more foxes are living off fewer rabbits when foxes have emotion, and both species having emotion lowers the combined population sizes. However, we also see in Fig. 4 that in the cases of higher fox population there is also a generally higher amount of predation of rabbits by foxes. Not only does this show that a high number of rabbits is not necessary to easily sustain the fox population, it also shows that the situation of only rabbit emotion allows rabbits to avoid foxes the best.

From analysis of these population changes we conclude that it is in the best interest of both species to use emotions. However, it is unclear whether this benefit derives from an individual benefit, or purely from the shown population benefit. These trends are essentially what we would expect to see if they were derived from individual utility, and thus we will examine individual benefits explicitly. Determining the level of effect of individual benefit will also lead to implications for the evolutionary aspect of emotion in this type of system.

\section{B. Individual Utility}

We next analyze the individual utilities for predator (fox) and prey (rabbit) for both utility functions to determine how individual utility relates to population gain. Overall the internal utility of individual predators does not change with

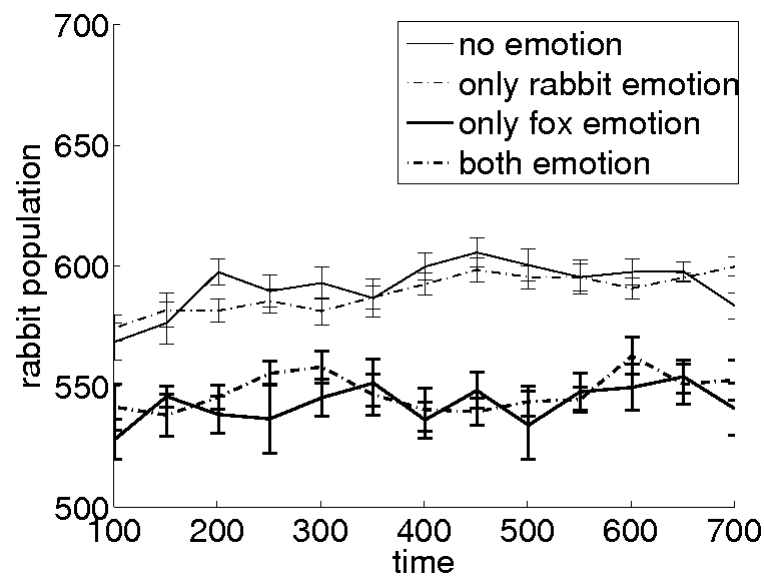

(a) Rabbit population dynamics

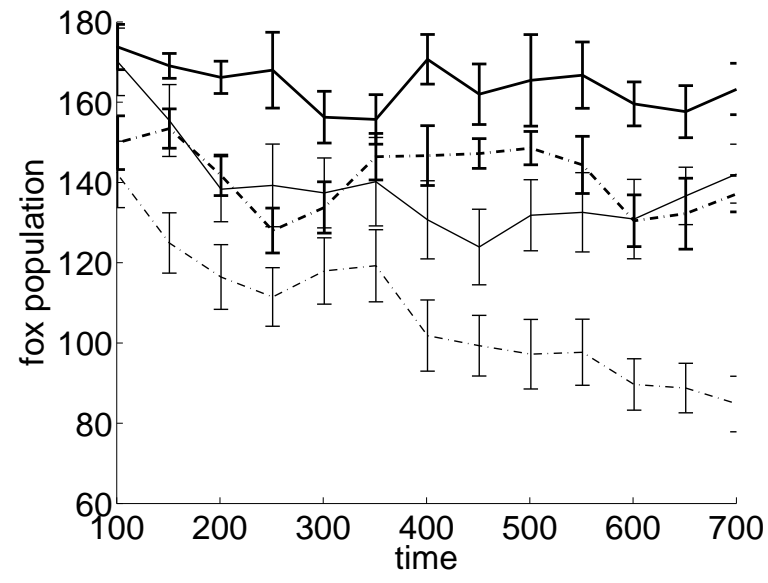

(b) Fox population dynamics.

Fig. 3: Population dynamics under the four emotion configurations: $3 \mathrm{a}$ the changes in rabbit population over time, and $3 b$ the changes in fox population over time.

respect to which species are using emotion (Fig. 5b). This is especially interesting as the predator population is affected by the use of emotion (Fig. 3b). Although the fox population as a whole is benefitted in the case where only foxes use emotion, and suffers when only rabbits use emotion, the individual is not significantly impacted by the change of emotional situation in terms of food intake. The internal utility for rabbits improves when only rabbits have emotion while it is degraded when only foxes have emotion (Fig. 5a), similar to what is seen in the rabbit population changes (Fig. $3 a)$. These results imply that rabbits benefit the most as both individuals and a population when foxes do not use emotion, and the least when only foxes use emotion. This improvement of internal prey utility when only the prey has emotion indicates that emotions and emotional communication are serving to assist in feeding, predation avoidance, and/or disease avoidance. While we have noted that when only predators have emotion predator utility does not improve, the prey utility decreases. This may be an indicator of predatorprey chases where the utility of the prey decreases due to the inability to feed, while the predator cannot eat until it 


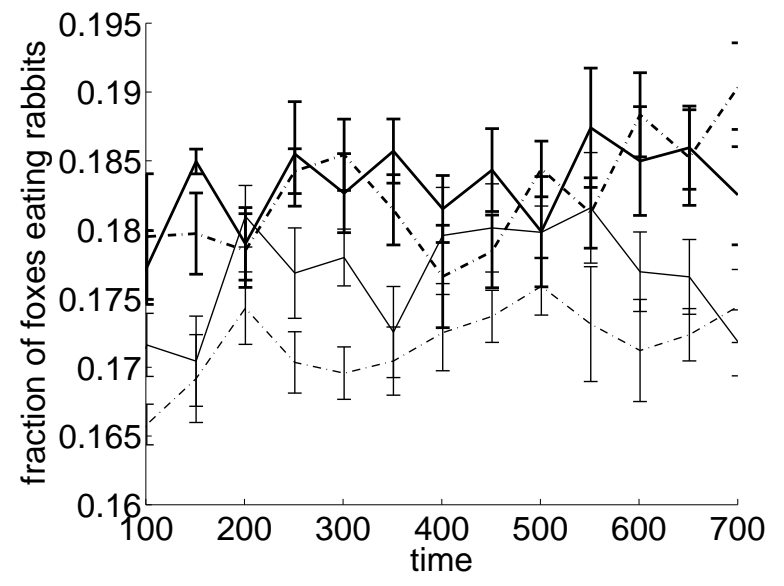

Fig. 4: Fraction of foxes eating rabbits.

catches the prey.

However, when both species have emotion the rabbit internal utility is indistinguishable from the condition when neither species has emotion. This suggests that in terms of the individual utility presented in Eq. 7 the use of emotion by both species may equally counteract the benefits and drawbacks of only rabbit and only fox emotion respectively. There is a distinguishable population increase when no emotion is used versus both species using emotion, however. This difference may lie in the fact that emotions are increasing reproduction, which does not affect the rabbit's internal utility.

The reproductive utility from Eq. 8 is based on the internal utility, but also decreases the longer a rabbit or fox exists without reproducing. As with the internal utility, the difference for prey based on emotion is much stronger than the difference for predators. However, the ordering of emotional configurations for rabbits is somewhat surprising. When only rabbits have emotion the reproductive utility is at its lowest, while it is at its highest when only foxes have emotion. This is directly opposed to what was seen with the internal utility. However, since in our model an individual's reproduction does not offer a direct benefit to the individual, this observation is not counter to our conclusion that individual prey utility improves with emotion. The lack of variability in both internal and reproductive predator utilities (Figs. $5 \mathrm{~b}$ and $6 \mathrm{~b}$ ) could be due to the longer predator lifespan or even the smaller number of predators that can be observed.

\section{DISCUSSION}

When relating individual utility to the population dynamics of rabbits the primary differences are the configurations when both species have emotion and neither species has emotion. As opposed to the population dynamics where both configurations behave similarly, both species with emotion correlates with only foxes having emotion and neither species having emotion correlates with only rabbits having emotion. The question this raises is how can individual utility decouple

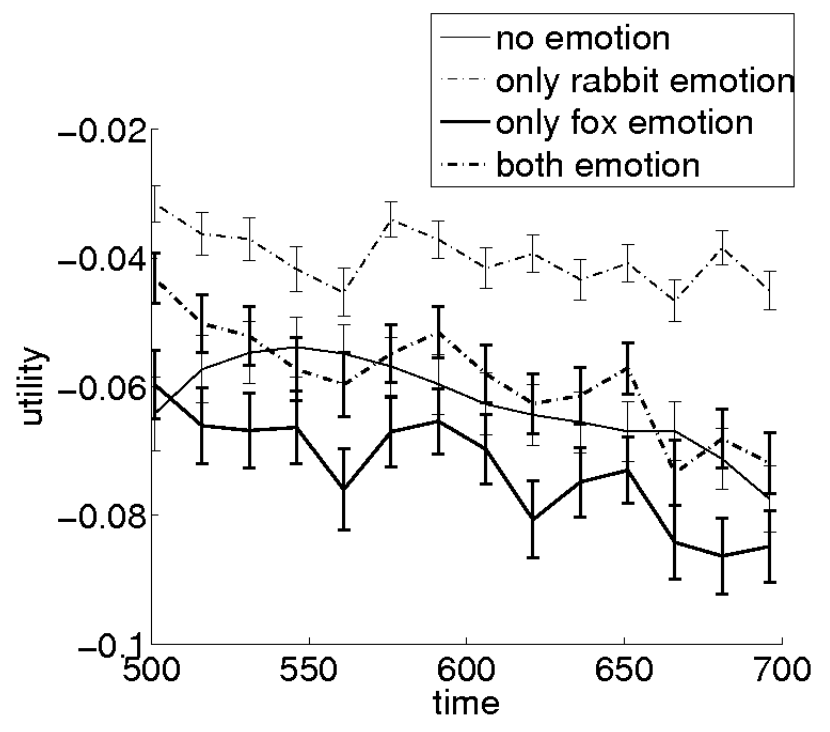

(a) Average individual rabbit utility.

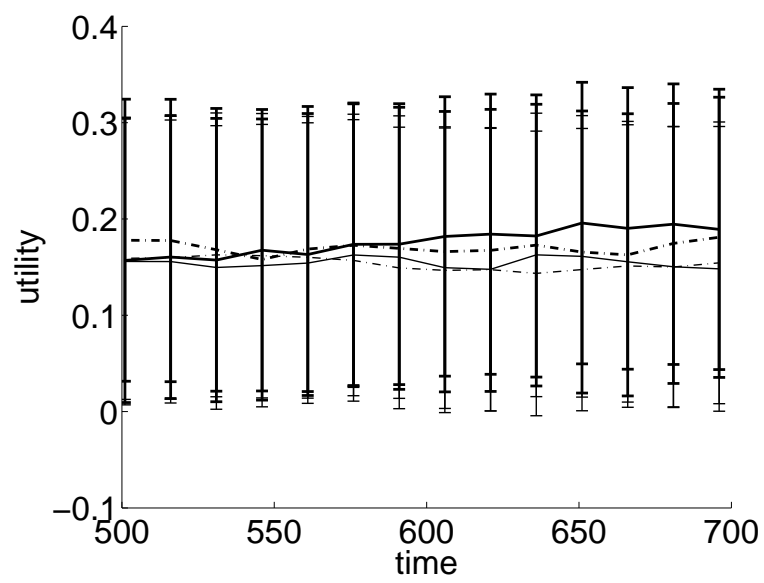

(b) Average individual fox utility.

Fig. 5: Rabbit and fox internal utilities under the 4 emotion configurations (Eq. 7).

from the global population dynamics? While we previously suggested that the correlation of rabbit population sizes between no emotion and only rabbit emotion may be due to a carrying capacity limiting the rabbit population size, this does not explain the population/individual bifurcation when both species have emotion and only foxes have emotion. This latter situation most probably stems from both the increased frequency of foxes eating rabbits and the increased fraction of diseased foxes. Foxes preying upon diseased rabbits will increase the average individual rabbit utility by removing diseased rabbits from the population.

To explain the results of individual reproductive utilities we relate back to the extended population dynamics study [23] where it was shown that the highest fraction of reproducing rabbits occurs when only foxes have emotion. This arises from the higher fraction of foxes that eat rabbits when only foxes have emotion. The low reproductive utility of rabbits when only rabbits have emotion can be explained similarly 


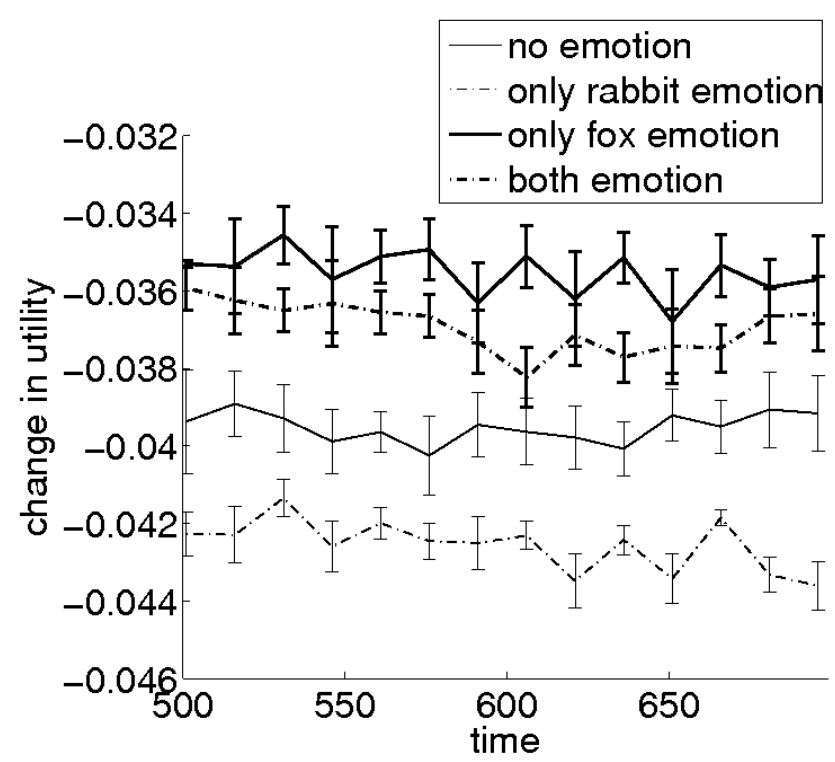

(a) Average change in individual rabbit reproductive utility.

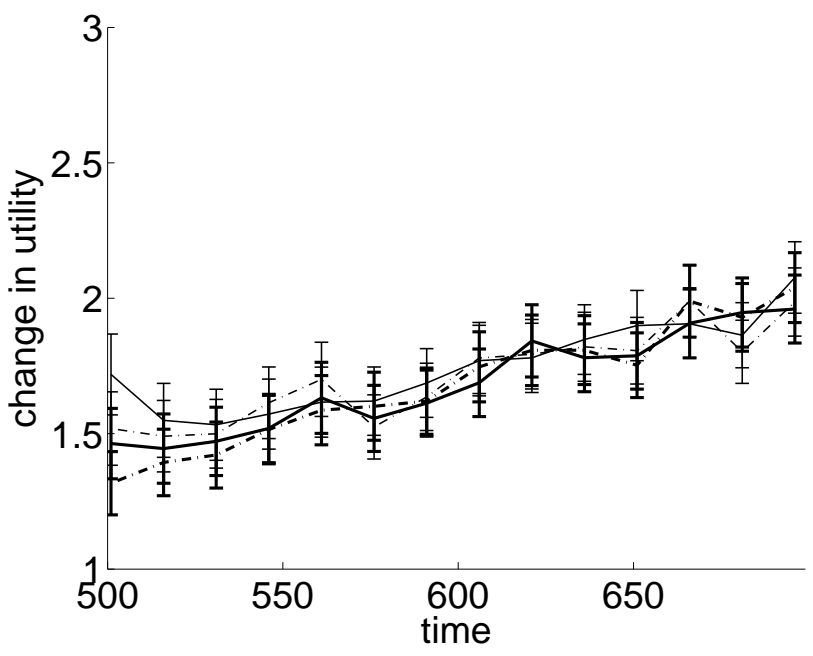

(b) Average change in individual fox reproductive utility.

Fig. 6: Change in rabbit and fox reproductive utilities under the 4 emotion configurations (Eq. 8).

by the observation that under this configuration the smallest fraction of rabbits reproduce. Yet we again see that trends in the utility for foxes are unclear.

Reproductive utility in foxes under all emotional configurations follow a similar trend. This result is similar to that shown in Fig. 5a. While the explanation for internal utility based upon the predation habits and disease frequencies in the fox population applies as well there is an upward trend in the change in utility. This is due to the oscillatory nature of the fox population. Growth rates for foxes tend to oscillate during a simulation. We suggest that the upward trend is due to a temporary increase in the growth rate of the fox population. It is interesting to note that the dynamics of average fox internal utility as well as the change in reproductive utility both offer no clear distinction between emotional configurations.
This model represents a subset of a larger food web which would involve many more interactions between entities; in particular, foxes would be subject to predation. The greater sensitivity of rabbit individual utility to change in emotion configuration may be due to the simulation of both rabbit prey and predators. On the other hand, fox individual utility appears to be insensitive to change in emotional configuration. However, the effect of changed emotion configuration on population dynamics is clear for both rabbit and fox.

The ordering of emotion configurations of fox population dynamics matches the ordering seen in both rabbit individual utilities. This suggests that both individuals and a species as a whole can benefit from the use of communicated somatic markers. As such, communicated somatic markers appear to promote cooperation between the individual and its population. The contribution of emotion to cooperative behavior is complex. Emotions such as anger, sadness, and disgust serve to push neighboring individuals away. Fear also has this effect, but it is coupled to the dynamics of the other species. Happiness is the only emotion that draws individuals together in our model, while surprise focuses the individual on more recent events. Each of these somatic markers provides key information for decisions of survival.

\section{CONCLUSION}

We have presented an analysis of individual versus species gain in a predator-prey system utilizing emotions. The relationship between individual and species utility suggests a mechanism by which communicated somatic markers can evolve with little or no individual cost and significant collective gain. This mechanism is an example of how altruistic behaviors such as cooperation [6] may evolve. When comparing individual utilities to population dynamics we observe some differences as well as some notable similarities. Fox utility does not informatively change with respect to emotion configuration. We suggest this is because the model does not represent a full food web with predator-prey relationships for each species. Both measures of individual rabbit utility resemble the population dynamics. Similarity in individual utility and population dynamics suggests that the mechanism of communicated somatic markers can promote cooperation within a species. We propose that the underlying phenomena is a result of emotional communication. Counter-arguments can be made that the observed differences arise from the dynamics of entity interactions and disease within the simulation. Further studies are required to invalidate these possibilities; however, our observations of multiple utility measures lend credence to the idea that individual emotional behaviors can offer collective gains while improving individual utility.

The cooperative gains of communicated somatic markers are clearly shown for the modeled rabbit species. However, the question of optimal behavior in a heterogeneous model may have alternative results. Parasitic behaviors would manifest as non-transmission or false transmission of somatic signals. We expect that although both behaviors may be present amongst cooperators in a mixed population, neither behavior is sustainable. Non-communicating entities may 
gain an edge over cooperative entities under some situations such as fleeing, but cooperative entities communicate reproductive and coordination signals, which may benefit most participants. Lying parasitism would probably have detrimental effects as lies would be encoded in the memory of neighbors as truth, which would then be interpreted as truth by the liars themselves. Game theoretic models of cooperation lack the spatial dimensions and interactive multi-agent dynamics offered by our model, making this observation of cooperation via both individual and population benefit significant.

Somatic markers provide key information about an individual's state that, when communicated, promote cooperation. These cooperative gains suggest that there is not a clear dichroic spectrum between individualist and collectivist behaviors. That is, in some situations it is possible to improve an individual's utility without cost to the population, and vice versa. While there are open arguments about what is the fundamental unit of selection in evolution [15], there is no doubt that the individual entity plays a significant role in evolutionary selection. By suggesting that under certain conditions the communication of somatic markers may not be at the expense of the individual it seems plausible that evolution operating on individuals may lead to collective improvements for a species. This conclusion is supported by studies that suggest innate encoding of emotion such as Ekman et al.'s pan-cultural facial emotion studies [16]. Although we acknowledge that many factors contribute to evolutionary selection pressures, we make a point to note that the species studied by Ekman et al. has continued to show improvement in terms of population dynamics.

\section{FUTURE WORK}

Recent studies have begun to understand social behaviors at a genetic level through coevolution [11]. Future work should consider the coevolution of somatic markers within a social model such as the one presented in this work.

\section{REFERENCES}

[1] R. Adolphs, D. Tranel, and A.R. Damasio. The human amygdala in social judgment. Nature, 393(6684):470-474, 1998.

[2] R.D. Alexander. The evolution of social behavior. Annual Review of Ecology and Systematics, 5:325-383, 1974.

[3] D.G. Amaral. The amygdala, social behavior, and danger detection. Annals of the New York Academy of Sciences, 1000(Emotions Inside Out: 130 Years after Darwin's The Expression of the Emotions in Man and Animals):337-347, 2003.

[4] M.A. Arbib and J.M. Fellous. Emotions: from brain to robot. Trends in cognitive sciences, 8(12):554-561, 2004.

[5] R.C. Arkin. Moving up the food chain: Motivation and Emotion in behavior-based robots. Who Needs Emotions: The Brain Meets the Robot, pages 245-270, 2005.

[6] R. Axelrod and W.D. Hamilton. The evolution of cooperation. Science, 211(4489):1390, 1981.

[7] J. Bachevalier and K.A. Loveland. The orbitofrontal-amygdala circuit and self-regulation of social-emotional behavior in autism. Neuroscience \& Biobehavioral Reviews, 30(1):97-117, 2006.

[8] A. Bechara, H. Damasio, and A.R. Damasio. Emotion, decision making and the orbitofrontal cortex. Cerebral cortex, 10(3):295, 2000.

[9] A. Bechara, H. Damasio, A.R. Damasio, and G.P. Lee. Different contributions of the human amygdala and ventromedial prefrontal cortex to decision-making. The Journal of Neuroscience, 19(13):5473, 1999.
[10] J.S. Beer, O.P. John, D. Scabini, and R.T. Knight. Orbitofrontal cortex and social behavior: integrating self-monitoring and emotion-cognition interactions. Journal of Cognitive Neuroscience, 18(6):871-879, 2006.

[11] J.Y. Chiao and K.D. Blizinsky. Culturegene coevolution of individualismcollectivism and the serotonin transporter gene. Proceedings of the Royal Society B: Biological Sciences, 277(1681):529, 2010.

[12] A.R. Damasio. Looking for Spinoza: Joy, sorrow, and the feeling brain. Mariner Books, 2003

[13] A.R. Damasio, BJ Everitt, and D. Bishop. The somatic marker hypothesis and the possible functions of the prefrontal cortex [and discussion]. Philosophical transactions: Biological sciences, 351(1346):1413-1420, 1996.

[14] C. Darwin, P. Ekman, and P. Prodger. The expression of the emotions in man and animals. Oxford University Press, USA, 2002.

[15] R. Dawkins. The selfish gene. Oxford University Press, USA, 2006.

[16] P. Ekman, E.R. Sorenson, and W.V. Friesen. Pan-cultural elements in facial displays of emotion. Science, 164(3875):86, 1969.

[17] S.C. Gadanho and J. Hallam. Robot learning driven by emotions. Adaptive Behavior, 9(1):42, 2001.

[18] W. James. The physical basis of emotion. Psychological Review, 1(5):516-529, 1894.

[19] C.P. Lee-Johnson and D.A. Carnegie. Emotion-based parameter modulation for a hierarchical mobile robot planning and control architecture. In Intelligent Robots and Systems, 2007. IROS 2007. IEEE/RSJ International Conference on, pages 2839-2844. IEEE, 2007.

[20] C.P. Lee-Johnson and D.A. Carnegie. Robotic Emotions: Navigation with Feeling. Handbook of Research on Synthetic Emotions and Sociable Robotics: New Applications in Affective Computing and Artificial Intelligence, page 88, 2009.

[21] Megan M Olsen, Kyle I Harrington, and Hava T Siegelmann. Computational Emotions Encourage Collective Behavior in Population Dynamics. In Proc. of 8th International Conf. on Complex Systems, 2011.

[22] M.M. Olsen, K. Harrington, and H.T. Siegelmann. Emotions for Strategic Real-Time Systems. In AAAI Emotion, Personality, and Social Behavior Technical Report, pages 104-110. Março, 2008.

[23] M.M. Olsen, K.I. Harrington, and H.T. Siegelmann. Conspecific Emotional Cooperation Biases Population Dynamics: A Cellular Automata Approach. International Journal of Natural Computing Research (IJNCR), 1(3):51-65, 2010

[24] D. Tranel, A. Bechara, and N.L. Denburg. Asymmetric functional roles of right and left ventromedial prefrontal cortices in social conduct, decision-making, and emotional processing. Cortex, 38(4):589-612, 2002.

[25] S. Wolfram and M. Gad-el Hak. A new kind of science. Applied Mechanics Reviews, 56:B18, 2003. 